\title{
Recombinant protein rMBP-NAP restricts tumor progression by triggering antitumor immunity in mouse metastatic lung cancer
}

\begin{tabular}{|r|l|}
\hline Journal: & Canadian Journal of Physiology and Pharmacology \\
\hline Manuscript ID & cjpp-2017-0186.R1 \\
\hline Danuscript Type: & Article \\
\hline Complete List of Authors: & $\begin{array}{l}\text { Wang, Ting; Zhengzhou University, School of life sciences } \\
\text { Du, Mingxuan; Zhengzhou University, School of life sciences } \\
\text { Ji, Zhenyu; Henan Academy of Medical and Pharmaceutical Sciences } \\
\text { Ding, Cong; Zhengzhou University, School of life sciences } \\
\text { Wang, Chengbo; Zhengzhou University, School of life sciences } \\
\text { Men, Yingli; Zhengzhou University, School of life sciences } \\
\text { Liu, Shimeng; Zhengzhou University, School of life sciences } \\
\text { Liang, Taotao; Zhengzhou University, School of life sciences } \\
\text { Liu, Xin; Zhengzhou University, School of life sciences } \\
\text { Kang, Qiaozhen; Zhengzhou University, School of life sciences }\end{array}$ \\
\hline Is the invited manuscript for \\
consideration in a Special \\
Issue?:
\end{tabular}$\quad$\begin{tabular}{l} 
N/A \\
\hline Keyword:
\end{tabular}

\section{SCHOLARONE \\ Manuscripts}




\section{Recombinant protein $\mathrm{rMBP}-\mathrm{NAP}$ restricts tumor progression by triggering antitumor immunity in mouse metastatic lung cancer}

Ting Wang ${ }^{1, \#, ~ M i n g x u a n ~} \mathrm{Du}^{1, \#}$, Zhenyu $\mathrm{Ji}^{2}$, Cong Ding ${ }^{1}$, Chengbo Wang ${ }^{1}$, Yingli Men ${ }^{1}$, Shimeng Liu ${ }^{1}$, Taotao Liang $^{1}$, Xin Liu $^{1, *}$, Qiaozhen Kang ${ }^{1, *}$

${ }^{1}$ School of Life Sciences, Zhengzhou University, No.100 Science Avenue, Zhengzhou 450001, P. R. China

${ }^{2}$ Henan Academy of Medical and Pharmaceutical Sciences, Zhengzhou University, 40 University Road, Zhengzhou 450052, P.R. China

${ }^{*}$ Corresponding author: Prof. Qiaozhen Kang and Dr. Xin Liu E-mail: qzkang@zzu.edu.cn; Liux@zzu.edu.cn

Tel: +8637167783236

Fax: +8637167783235

\# The authors contributed equally to this work. 


\begin{abstract}
Recombinant Helicobacter pylori neutrophil-activating protein fused with Maltose-binding protein (rMBP-NAP), a potential TLR2 ligand, was reported to possess immunomodulatory effects on in-situ tumor in our previous study. In present work, we attempt to elucidate the effect of rMBP-NAP at the local immune modulation in B16-F10-induced metastatic lung cancer. Our results demonstrated that growth of B16-F10 melanoma metastases in the lung was significantly arrested after rMBP-NAP treatment, along with marked reduction in metastatic lung nodules and significant increase in survival. The treatment induced both local and systemic immune responses, which associated with higher influx of $\mathrm{CD} 4^{+} / \mathrm{CD} 8^{+} \mathrm{T}$ cells and drove toward Th1-like and cytotoxic immune environment. Moreover, rMBP-NAP also showed significant anti-angiogenic activity by reducing vascularization in lung tumor sections. In conclusion, rMBP-NAP could induce antitumor immunity through activating Th1 cells and producing pro-inflammatory cytokines, which are responsible for the effective cytotoxic immune response against cancer progression. Our findings indicate that rMBP-NAP might be a novel antitumor therapeutic strategy.
\end{abstract}

Keywords: rMBP-NAP; Metastatic lung cancer; Toll-like receptor; Antitumor immunotherapy; Immunomodulator 


\section{Introduction}

Metastatic lung cancer is one of the principal causes of death. It is a low immunogenic cancer, resistant to the surveillance of the immune system (Pinto et al. 2011; Igney and Krammer 2002). Despite advances in treatment, the prognosis remains poor 5 years survival benefit. Although, in the past 20 years, the Food and Drug Administration has approved the systemic administration of interferon (IFN)- $\alpha$ for patients in stage II or III with high risk of metastatic disease, the stimulating interest is still focus on development of new therapeutic strategies (de Melo et al. 2015). The terminology of "cancer immunoediting" has been used to describe the process of shaping the immunogenicity of tumors, which relies upon the activation of the adaptive immune system to recognize and eliminate "transformed" cells (Prendergast 2008; Forte et al. 2012). However, the tumor microenvironment is characterized by a process of chronic inflammation, which can facilitate either tumor establishment or progression (Colotta et al. 2009). Therefore, the activation of the immune system could represent a means to induce tumor regression (Igney and Krammer 2002; Prendergast 2008).

Over a century ago, Dr. William Coley injected microorganisms that we now know include a number of Toll-like receptor (TLR) agonists into tumors and observed systemic antitumor responses (Mellman et al. 2011; Takeda et al.2003; Liew et al. 2005). Following activation by ligands, TLRs can enhance the uptake of pathogens by phagocytic cells and also mediate leukocyte recruitment to pathologic focus through secreting multiple cytokines and chemokines (Mogensen 2009; Hajishengallis and Lambris 2011). On the other hand, TLRs also express on adaptive immune cells and modulate the correlation between subsets of $\mathrm{T}$ cells and innate immune cells (Caron et al. 2005; Zhang et al. 2011). Based on the broad immune 
activation effect, TLR agonists become attractive candidates for adjuvants of cancer immunotherapy.

Increasing evidence suggests that TLRs are important regulators of tumor biology, however, little is known about their function in metastatic lung cancer. In order to understand the connection between TLRs and metastatic lung cancer progression, several TLR agonists are currently being tested as immunomodulator for anticancer therapies (Pinto et al. 2011; Forte et al. 2012). The TLR2 ligand, Helicobacter pylori neutrophil-activating protein (Hp-NAP), has been shown to possess a potential role as biological response modifier. Hp-NAP is a key factor driving Th1 inflammation in H. pylori infection. Furthermore, recombinant Hp-NAP itself could serve as a potential drug candidate in immunotherapy of cancer (Codolo et al. 2012; Iankov et al. 2012; Ramachandran et al. 2013). Our previous study demonstrated that the recombinant Hp-NAP fused with the maltose-binding protein of Escherichia coli (rMBP-NAP), possesses a significant immunomodulating effects on in-situ tumor, rather than direct killing of tumor cells (Wang et al. 2015).

The present study is focused on the effect of rMBP-NAP that acts as an immunomodulator in the experimental mouse model of B16F10 melanoma lung metastasis. We show that rMBP-NAP can induce both local and systemic immune responses, which associated with higher influx of $\mathrm{CD}^{+} \mathrm{CD}^{+} \mathrm{T}$ cells, $\mathrm{CD}^{+} \mathrm{CD} 8^{+} \mathrm{T}$ cells and drove toward a Th1-like and cytotoxic immune environment to tumor, with higher secreted interferon (IFN)- $\gamma$ and interleukin (IL)-27 cytokines in lung tissue and spleen. Moreover, rMBP-NAP also showed significant anti-angiogenic activity by reducing vascularization in tumor section of lung in B16F10-bearing mouse. In conclusion, our study proves rMBP-NAP therapeutic efficacy via 
the activation and maintenance of T cells immunity, the production of Th1-like cytokines and induction of anti-angiogenesis effect, which in turn facilitates efficient anticancer activity in a mouse model of metastatic lung cancer. 


\section{Material and methods}

\section{Animals and cell lines}

Animals were handled by specialized personnel in Henan Academy of Medical and Pharmaceutical Science (Certificate No. SCXK (Yu) 2011-0010, Henan, China). All procedures are specified in the project approved by the University committee for animal experiments. C57BL/6J mice, aged 6-8 weeks (initial weight $18 \pm 2 \mathrm{~g}$ ), were purchased from Vital River Laboratories Animal Technology Co., Ltd. (Certificate No. SCXK (Jing) 20120001, Beijing, China). The B16-F10 mouse melanoma cell line was maintained in DMEM medium supplemented with $10 \%$ heat-inactivated fetal bovine serum at $37^{\circ} \mathrm{C}$ in humidified $5 \%$ $\mathrm{CO}_{2}$.

\section{Mice treatment}

Mice were injected (i.v.) with $2 \times 10^{5}$ B16-F10 cells (day 1 ), and after $24 \mathrm{~h}$ rMBP-NAP $(0.5$, 2.5 , or $5 \mathrm{mg} / \mathrm{kg}$; 6 mice each group) was administered by the i.p. route for 7 times once every two days. Mice were administrated saline or cisplatinum (2 mg/kg; Sigma-Aldrich, Rome, Italy) as control group $(n=6)$ and positive control group $(n=6)$. Mice were euthanized 2 weeks after melanoma cells injection, the lungs were excised, and the pulmonary metastatic tumors were counted in a blind manner under a dissecting microscope.

For survival analysis, animals $(n=5)$ were treated with 10 consecutive doses of the protein prior to tumor inoculation and were injected intravenously with $2 \times 10^{5}$ B16-F10 cells after rMBP-NAP treatment. We registered the day of death for each mouse and these data were used to Kaplan-Meier survival analysis.

\section{Preparation of fusion protein rMBP-NAP}


The involved experiment procedures were all based on our previous published works (Kang et al. 2005). Briefly, neutrophil-activating protein gene of H pylori (Hp-napA) was subcloned from the recombinant plasmid pNEB-napA (restored in our laboratory), digested with EcoR I and Sal I (Takara, Japan) and inserted into the pMAL-c2x (New England Biolabs, Inc, USA) vector which prepared with the same enzymes to make pMAL-c2x-napA. The sequence confirmed plasmids were transformed into the E.coli strain TB1 (Invitrogen). The transformed cells were grown in LB medium containing $50 \mu \mathrm{g} / \mathrm{ml}$ kanamycin in $100 \mathrm{ml}$ batches at $37{ }^{\circ} \mathrm{C}$. IPTG was added to the medium to a final concentration of $0.3 \mathrm{mM}$ when the $\mathrm{OD}_{600}$ of the culture reached 0.5 . The expression of the fusion proteins was allowed for $3 \mathrm{~h}$ at $37{ }^{\circ} \mathrm{C}$. The cells were harvested and frozen at $-20{ }^{\circ} \mathrm{C}$ overnight, sonicated in ice-water bath, centrifuged at $9000 \mathrm{rpm}$ for $30 \mathrm{~min}$ at $4{ }^{\circ} \mathrm{C}$. Soluble rMBP-NAP in the supernatant was purified by amylose affinity chromatography. Endotoxin was removed with agarose bed columns containing immobilized polymyxin B (Thermo scientific, USA).

\section{Polymerase chain reaction (PCR)}

Total RNA was extracted with Trizol ${ }^{\circledR}$ (Invitrogen, USA) according to the manufacturer's instructions. Homogenize tissue samples in Trizol ( $1 \mathrm{ml}$ per $50 \mathrm{mg}$ of tissue) in appropriate homogenizer. Add $0.2 \mathrm{ml}$ of chloroform per $\mathrm{ml}$ of Trizol used. Centrifuge the resulting mixture at $12,000 \mathrm{rpm}$ for 15 minutes at $2-8{ }^{\circ} \mathrm{C}$. Transfer the aqueous phase to a fresh tube and add $0.5 \mathrm{ml}$ of 2-propanol. Centrifuge and remove the supernatant, then wash the RNA pellet by adding a minimun of $1 \mathrm{ml}$ of $75 \%$ ethanol and centrifuge at $12000 \mathrm{rpm}$ for 5 minutes at 2-8 ${ }^{\circ} \mathrm{C}$. Briefly dry the RNA pellet by air-drying. Total RNA was dissolved in RNase-free water and prepared for qRT-PCR analysis. 
Quantitative real time PCR was performed using the SYBR Premix Ex TaqTM II (Takara, Japan). Data was acquired using a LightCycler 1.5 System (ligheCycler, German). Cycle threshold values were normalized to amplification of $\beta$-actin. Relative quantification of gene expression levels was calculated using the $2^{-\Delta \Delta \mathrm{Ct}}$ methods. A list of the qRT-PCR primers used in the present study was given in suppl. Table S1.

\section{Isolation of splenocytes, lymphocytes and PBMC}

Spleens from individual mice were removed and disrupted by scratching in a sterile manner, Cells suspended in PBS were centrifuged at $1000 \mathrm{rpm}$ for 5 minutes and red blood cell osmotic lysis was carried out after incubation with $10 \mathrm{ml}$ of QB LysingTM Solution Buffer (Quantobio, China). Centrifuging and removing the supernatant, the cell pellet was resuspended in $2 \mathrm{ml}$ of RPMI-1640 medium and the viable splenocytes were counted by Trypan blue exclusion.

Lungs were removed, minced and incubated at $37^{\circ} \mathrm{C}$ for $45 \mathrm{~min}$ in PBS containing $1 \mathrm{mg} / \mathrm{ml}$ collagenase D and $0.2 \mathrm{mg} / \mathrm{ml}$ DNase I (Sigma-Aldrich, USA). After enzyme treatment, lung tissue was gently passed through a cell strainer $(70 \mu \mathrm{m}, \mathrm{BD}$ falcon, USA) and then washed twice in PBS. Cells were resuspended in 35\% Percoll (GE Healthcare, USA) in PBS, layered over $70 \%$ Percoll, and spun at 2,500 rpm for $20 \mathrm{~min}$. Lymphocytes collected from the interface were used for subsequent flow cytometry analysis.

Peripheral blood was collected by orbital sinus bleeding in a tube containing citrate sodium buffer. Anti-coagulated blood was layered onto Histopaque-1083 (Sigma-Aldrich, USA) and peripheral blood mononuclear cells $(\mathrm{PBMC})$ were purified by gradient centrifugation according to the manufacturer's recommendations. 


\section{IFN- $\gamma$ ELISPOT assay}

IFN- $\gamma$-secreting splenocytes were enumerated using enzyme-linked immunospot (ELISPOT) (Lycke and Coico 1996). Briefly, the splenocytes $\left(1 \times 10^{7}\right.$ cells $\left./ \mathrm{ml}\right)$ obtained from rMBP-NAP and PBS administrated tumor-bearing mice, were added to Immunospot plates (Dakewe Biotech, China), which precoated with an anti-IFN- $\gamma$ monoclonal antibody (mAb). Then, rMBP-NAP or PBS was added respectively. The positive control well was added PHA (Sigma-Aldrich, USA). The plates were incubated overnight at $37^{\circ} \mathrm{C}$ under humidified air with $5 \% \mathrm{CO}_{2}$. The wells were washed with deionized water and wash buffer and then incubated with biotin-conjugated anti-IFN- $\gamma \mathrm{mAb}$ at $37^{\circ} \mathrm{C}$ for $1 \mathrm{~h}$. After washes, streptavidin-alkaline phosphatase was added and the plates were incubated for $1 \mathrm{~h}$ at room temperature. The plates were again washed and added activator solution (Dakewe Biotech, China) to develop spots, then washed with distilled water to stop the reaction. Air dry completely at room temperature. Experiments were performed in triplicate. The number of spots in each well was counted using the BioReader 4000 Pro-X.

\section{Cytotoxicity assay}

The B16-F10 cells $\left(0.5 \times 10^{4}\right)$ in DMEM were used as target cells and incubated with PBMC, obtained from rMBP-NAP and PBS treated mice, for $4 \mathrm{~h}$ at $37{ }^{\circ} \mathrm{C}$ in a humidified atmosphere containing $5 \% \mathrm{CO}_{2}$. The cytotoxicity was determined by $\mathrm{LDH}$ release assay using a commercially available kit (Promega, USA). The percentage of lysis was calculated by the formula: \% Specific lysis $=($ Experimental lysis - Spontaneous lysis $/$ Maximum lysis Spontaneous lysis) $\times 100 \%$.

\section{Flow cytometry}


Freshly isolated splenocytes $\left(1 \times 10^{6}\right.$ cells $\left./ \mathrm{ml}\right)$ were incubated for $30 \mathrm{~min}$ at $4{ }^{\circ} \mathrm{C}$ with FITC conjugated anti-CD3, PE conjugated anti-CD4 or PerCP conjugated anti-CD8 (eBioscience, USA). Antibody labeling was performed via a standard procedure. Flow cytometry analysis was carried out by using BD FACSCalibur cell analyzer (BD Biosciences, USA) and the data was analyzed with the FlowJo software.

\section{Immunohistochemistry staining}

Immunohistochemical studies were performed on 5-mm-thick sections prepared from formalin-fixed and paraffin-embedded tissue using standard staining protocols. Deparaffinization and antigen retrieval were carried out as a standard procedure. Microvessel density (MVD) represented by CD34 staining was measured according to the methods (Weidner et al. 1991). The resultant sections were first examined at low magnifications $(4 \times)$ to identify the vascular-rich area in the tumor. Within this area, the CD34-positive microvessels were counted in a single high-power $(20 \times)$ field. Any CD34 stained single or cluster of cells was considered a single countable microvessel.

\section{Statistics}

Results are expressed as means \pm SEM. Changes observed in treated groups compared with controls were analyzed using GraphPad Prism 5.01 followed by unpaired two-tailed Student's $\mathrm{t}$ test, and/or two-way ANOVA where appropriate. The $p$ values $<0.05$ was considered to be statistically significant. 


\section{Results}

\section{rMBP-NAP decreases lung metastatic tumor growth and prolongs survival of mice}

The immunotherapeutic effect of the association of rMBP-NAP was evaluated in the metastatic B16F10 melanoma model. The challenge with viable B16F10 tumor cells occurred on day $1, \mathrm{C} 57 \mathrm{Bl} / 6$ mice were treated with seven intraperitoneal inoculations of rMBP-NAP at one day intervals after the tumor bearing. 24 hours after the last treatment, mice were killed for lung nodules quantification and/or immunological assays (Fig. 1A).

Administration of rMBP-NAP promoted a marked reduction in the number of lung nodules in B16F10 injected mice (Fig. 1B). Lung metastasis frequencies significantly were reduced by $43.9 \%, 71.9 \%$, and $56.9 \%$ in rMBP-NAP treated mice at dosage of $0.5 \mathrm{mg} / \mathrm{kg}, 2.5 \mathrm{mg} / \mathrm{kg}$, $5 \mathrm{mg} / \mathrm{kg}$, respectively, and the number of lung foci in cisplatinum-treated group was reduced to 41.6\%, compared with that of PBS-treated mice (Fig. 1C). According to the results above the dose of $2.5 \mathrm{mg} / \mathrm{kg}$ of rMBP-NAP was chosen in the following assays. The weight of lung tissues was markedly reduced in response to rMBP-NAP vs PBS control, while no significant change in body weight (Fig. 1D and 1E). For survival analysis, mice inoculated with rMBP-NAP showed a prolonged survival in comparison to animals treated with PBS (Fig.1F). This observation indicates that rMBP-NAP treatment reduces development of metastatic nodules in the lungs.

rMBP-NAP induces anti-tumor immune response against B16-F10 tumor cells in the spleen.

As we known, a Th2 cytokine profile is associated with down-regulation of the immune response and progression of melanoma. The polarization of Th1 cells is thought to contribute 
to cellular immune response and increase $\mathrm{CD} 8^{+}$cytotoxic $\mathrm{T}$ lymphocytes. The cytokine profiles in spleen were measured by ELISPOT and qRT-PCR assay. IFN- $\gamma$-secreting cells were obviously increased after treated with rMBP-NAP (Fig. 2A). As shown in Fig. 2B, the expression of Th1 representative cytokines, IL-12 and IL-27, were effective elevated, the typical Th2 cytokine IL-4 expression was dramatically reduced in the rMBP-NAP treated mice, which indicated a Th1-dominanted milieu. To understand whether the immunomodulatory role of rMBP-NAP can elicit systemic $\mathrm{T}$ cytotoxic $(\mathrm{Tc}) 1$ immune response, the cytotoxicity of splenic cells and PBMC towards B16-F10 cells was assessed after rMBP-NAP treatment. The results showed when an effector/target ratio (E/T ratio) of 10:1 was used, the cytotoxic efficacy of PBMC cells and splenic lymphocytes from rMBP-NAP treated mice were remarkably augmented compared with control mice (Fig. 2C).

\section{rMBP-NAP can exhibit the immune regulatory effect in the tumor microenvironment}

It is becoming clear that tumors can actively subvert the immune system through a variety of immune suppressive mechanisms within the tumor microenvironment. To verify the cytokine profiles induced by rMBP-NAP in the tumor microenvironment, we performed a quantification of cytokines in mice lung homogenates. To this end, lung tissues from rMBP-NAP treated or untreated mice were excised. As shown in Fig. $3 \mathrm{~A}$ and $3 \mathrm{~B}$, the expression of Th1-like cytokine profiles such as IFN- $\gamma$ and IL27 were significantly increased. In other hand, the mRNA expression of proinflammatory cytokines (IL-6, TGF- $\beta$ ) and chemokines (CCL2、CCL20) were remarkably decreased (shown in Fig. 3C). To verify the role of $\mathrm{T}$ lymphocytes in the protective antitumor effect induced by rMBP-NAP, we examined the types of infiltrating cells within the lung tissue and found that $\mathrm{CD} 3^{+} \mathrm{CD} 8^{+} \mathrm{T}$ cells were 
significantly augmented after treatment (Fig.3D). In addition, the adhesion molecules ICAM-1, and VCAM-1 which is important regulator to the recruitment of effector T lymphocyes, were significantly enhenced compared with control (Fig.3E). These data indicated that rMBP-NAP has potential activity to regulate the tumor immunosuppressive microenvironment and enhance the adaptive cell immunity.

\section{rMBP-NAP decreases VEGF levels in the lungs of tumor-bearing mice}

To explore the involvement of suppression of angiogenesis in the antitumor activity induced by rMBP-NAP, we evaluated the inhibitory effect of rMBP-NAP on tumor-induced neovascularization by immunohistochemical staining of lung tissue section with anti-CD34 antibody. As shown in Fig. 4A, the microvessel which stained by anti-CD34 is much less in rMBP-NAP treated mice compared with control. Furthermore, qRT-PCR analysis also showed the production of antiangiogenic cytokines, VEGF-A and VEGF-D, were decreased in the tumor sections with rMBP-NAP treatment (Fig. 4B and 4C). 


\section{Discussion}

Immunotherapy is emerging as a powerful and active tumor-specific approach against cancer via triggering the immune system. TLR agonists used as single agents can effectively eradicate tumors due to their potent stimulation of innate and adaptive immunity.

TLRs are fundamental elements of the immune system, which facilitate our understanding of the innate and adaptive immune pathways. TLR signaling could lead to tumor cell death, resulting in tumor regression or arrest via inducing Th1-like and cytotoxic immunity (Wang et al. 2006). Given that cancerous cells evade the immune system, the activation of TLRs could represent an efficient approach against cancer. Therefore, our currently study assessed the beneficial antitumor and anti-metastasis activity of rMBP-NAP on experimental pulmonary metastasis in mice.

The treatment with rMBP-NAP rendered protection against metastatic B16F10 melanoma by reducing the number of lung nodules and increasing the survival of treated mice. Nevala et al. demonstrated Th2 cytokine dominant systemic environment in patients with metastatic melanoma (Nevala et al. 2009). In current study, we showed that rMBP-NAP could stimulate IFN- $\gamma$ production in the spleen, promote the mRNA expression of IL12 and IL27 and reduce the expression of IL4, which profoundly stimulated systemic antitumor Th1 response by inducing Th1/Th2 balance drift toward Th1. Moreover, we demonstrated that the cytotoxic efficacy of PBMC cells and splenic lymphocytes from rMBP-NAP treated mice were augmented, compared with PBS control mice. Our findings strongly prompted that the tumoricidal activity induced by rMBP-NAP was due to its capacity to elicit systemic Th1 and T cytotoxic (Tc) 1 immune response. 
As we learn more about the processes that contribute to antitumor immune response, there is increased interest in altering the microenvironment directly in a manner that enhances cell-mediated immunity. Most recent investigation showed Th1 cells have an important role in cellular immune responses by secreting IFN- $\gamma$ and capable of enhancing activity of CD $8^{+}$ CTLs, traditionally been considered the most efficient $\mathrm{CD}^{+} \mathrm{T}$ cell subset to generate antitumor immunity (Knutson and Disis 2005). Indeed, upon manipulation of the melanoma microenvironment with rMBP-NAP, we observed a profound influx of both $\mathrm{CD} 3^{+} \mathrm{CD} 8^{+}$and $\mathrm{CD}^{+} \mathrm{CD}^{+}$effector $\mathrm{T}$ cells into the lung interstitium and increased levels of numerous Th1-like cytokines and inflammatory mediators (e.g., IFN- $\gamma$, IL12, IL27) in association with augmented amounts of Th1 cells and immunestimulative function, indicating an immunomodulatory effect of rMBP-NAP in melanoma leisions.

Tumor microenvironment is characterized by chronic inflammation represented by infiltrating leukocytes and soluble mediators, which lead to a local and systemic immunosuppression associated with cancer progression (Mantovani et al. 2008). The intraperitoneal administration of rMBP-NAP in mice promoted infiltration of lymphocyte in the lungs and reduced the local production of several proinflammatory cytokines (IL-6, TGF- $\beta$ ), indicating an anti-inflammatory effect in local area. Results from mRNA expression of chemokines consistently revealed that only rMBP-NAP treatment substantially decreased the expression of CCL2 and CCL20 in tumor tissues as compared with control mice. Chemokines CCL2 and CCL20 were also reported has capable to recruit MDSC and Regulatory T cells (Treg) to tumor microenviroment, which central to form immunosuppression (Borsig et al. 2014; Liu et al. 2015). These data together indicated that rMBP-NAP treatment induced different antitumor 
immune responses in the tumor tissues. Intraperitoneal administration induced lymphocyte infiltration and regulated the expression of inflammatory mediators, improving the clearance of tumor of the lungs and increasing the survival of treated.

Since Folkman first demonstrated the importance of angiogenesis in tumor initiation, development and metastasis (Folkman 1990), it was thought of one of promising targets for tumor therapy (Kerbel 2008; Streit and Detmar 2003). So much attention had been paid to angiogenesis is required for a variety of physiological and pathological processes. At present, we stained pulmonary sections for the vascular marker CD34. A clear decrease was observed in overall vascularization in rMBP-NAP treated mice, along with the reduced expression of antiangiogenic cytokines, VEGF-A and VEGF-D. In parallel, the high levels of IFN- $\gamma /$ IL27 could exert a strong antiangiogenic activity leading to a dramatic reduction of tumor vascularization (Yin et al. 2014; Murugaiyan and Saha 2013). Interestingly, we had currently shown that IL-27 was detected high increased level both in spleen and pulmonary tissue from rMBP-NAP treated-mice, suggesting that rMBP-NAP was capable of exerting an anti-angiogenic activity due to its capacity of creating an IFN- $\gamma /$ IL27-enriched milieu by acting on innate immune cells, which plays an important role in its antitumor and antimetastatic activities. Although in vivo studies in humans will be necessary to assess the therapeutic efficacy of rMBP-NAP, our present results provide the basis to innovative therapies for relieving of metastatic lung cancer. Clearly, further studies are required for the validation of the possibility that rMBP-NAP, other than inducing Th1 responses, may promote antigen-specific immunity or act as tumor vaccine adjuvant against tumor represents an interesting issue that deserves further investigation. 


\section{Acknowledgement}

This work was supported by the grants from the National Natural Science Foundation of China (Code: 81373119,81571526 and 81602537).

\section{Conflict of Interest}

The authors of this manuscript do not have any conflict of interest to disclose. 


\section{References}

Borsig, L., Wolf, M.J., Roblek, M., Lorentzen, A., and Heikenwalder, M. 2014. Inflammatory chemokines and metastasis--tracing the accessory. Oncogene, 33(25): 3217-3224.

Caron, G., Duluc, D., Frémaux, I., Jeannin, P., David, C., Gascan, H., et al. 2005. Direct stimulation of human T cells via TLR5 and TLR7/8: flagellin and R-848 up-regulate proliferation and IFN-gamma production by memory $\mathrm{CD}^{+} \mathrm{T}$ cells. J. Immunol. 175(3): $1551-1557$.

Codolo, G., Fassan, M., Munari, F., Volpe, A., Bassi, P., Rugge, M., et al. 2012. HP-NAP inhibits the growth of bladder cancer in mice by activating a cytotoxic Th1 response. Cancer Immunol. Immunother. 61(1): 31-40.

Colotta, F., Allavena, P., Sica, A., Garlanda, C., and Mantovani, A. 2009. Cancer-related inflammation, the seventh hallmark of cancer: links to genetic instability. Carcinogenesis, 30(7): 1073-1081.

Cottalorda, A., Verschelde, C., Marcais, A., Tomkowiak, M., Musette, P., Uematsu, S., et al. 2006. TLR2 engagement on CD8 $\mathrm{T}$ cells lowers the threshold for optimal antigen-induced T cell activation. Eur. J. Immunol. 36(7): 1684-1693.

de Melo, F.M., Braga, C.J., Pereira, F.V., Maricato, J.T., Origassa, C.S., Souza, M.F., et al. 2015. Anti-metastatic immunotherapy based on mucosal administration of flagellin and immunomodulatory P10. Immunol. Cell. Biol. 93(1): 86-98.

Folkman, J. 1990. What is the evidence that tumors are angiogenesis dependent? J. Natl. Cancer Inst. 82(1): 4-6.

Forte, G., Rega, A., Morello, S., Luciano, A., Arra, C., Pinto, A., et al. 2012. 
Polyinosinic-polycytidylic acid limits tumor outgrowth in a mouse model of metastatic lung cancer. J. Immunol. 188(11): 5357-5364.

Gelman, A.E., Zhang, J., Choi,Y., and Turka, L.A. 2004. Toll-like receptor ligands directly promote activated $\mathrm{CD}^{+}{ }^{+} \mathrm{T}$ cell survival. J. Immunol. 172(10): 6065-6073.

Hajishengallis, G., and Lambris, J.D. 2011. Microbial manipulation of receptor crosstalk in innate immunity. Nat. Rev. Immunol. 11(3): 187-200.

Iankov, I.D., Allen, C., Federspiel, M.J., Myers, R.M., Peng, K.W., Ingle, J.N., et al. 2012. Expression of immunomodulatory neutrophil-activating protein of Helicobacter pylori enhances the antitumor activity of oncolytic measles virus. Mol. Ther. 20(6): 1139-1147.

Igney, F.H., and Krammer, P.H. 2002. Immune escape of tumors: apoptosis resistance and tumor counterattack. J. Leukoc. Biol. 71(6): 907-920.

Kang Q.Z., Duan G.C., Fan Q.T., and Xi Y.L. 2005. Fusion expression of Helicobacter pylori neutrophil-activating protein in E.coli. World J Gastroenterol. 11(3):454-456.

Kerbel, R.S. 2008. Tumor angiogenesis. N. Engl. J. Med. 358(19): 2039-2049.

Knutson, K.L., and Disis, M.L. 2005. Tumor antigen-specific T helper cells in cancer immunity and immunotherapy. Cancer Immunol. Immunother. 54(8): 721-728.

Komai-Koma, M., Jones, L., Ogg, G.S., Xu, D., and Liew, F.Y. 2004. TLR2 is expressed on activated T cells as a costimulatory receptor. Proc. Natl. Acad. Sci. U.S.A. 101(9): 3029-3034.

Liew, F.Y., Xu, D., Brint, E.K., and O'Neil, L.A. 2005. Negative regulation of toll-like receptor-mediated immune responses. Nat. Rev. Immunol. 5(6): 446-458.

Liu, J.Y., Li, F., Wang, L.P., Chen, X.F., Wang, D., Cao, L., et al. 2015. CTL- vs Treg 
lymphocyte-attracting chemokines, CCL4 and CCL20, are strong reciprocal predictive markers for survival of patients with oesophageal squamous cell carcinoma. Br. J. Cancer, 113(5): 747-755.

Lycke, N., and Coico, R. 2015. ELISPOT Assay for Measurement of Antigen-Specific and Polyclonal Antibody Responses. Curr. Protoc. Immunol. 108: 7 14 11-10.

Mantovani, A., Allavena, P., Sica, A., and Balkwill, F. 2008. Cancer-related inflammation. Nature, 454(7203): 436-444.

Mellman, I., Coukos, G., and Dranoff, G. 2011. Cancer immunotherapy comes of age. Nature, 480(7378): 480-489.

Mogensen, T.H. 2009. Pathogen recognition and inflammatory signaling in innate immune defenses. Clin. Microbiol. Rev. 22(2): 240-273.

Murugaiyan, G., and Saha, B. 2013. IL-27 in tumor immunity and immunotherapy. Trends Mol. Med. 19(2): 108-116.

Nevala, W.K., Vachon, C.M., Leontovich, A.A., Scott, C.G., Thompson, M.A., Markovic, S.N., et al. 2009. Evidence of systemic Th2-driven chronic inflammation in patients with metastatic melanoma. Clin. Cancer Res. 15(6): 1931-1939.

Pinto, A., Morello, S., and Sorrentino, R. 2011. Lung cancer and Toll-like receptors. Cancer Immunol. Immunother. 60(9): 1211-1220.

Prendergast, G.C. 2008. Immune escape as a fundamental trait of cancer: focus on IDO. Oncogene, 27(28): 3889-3900.

Ramachandran, M., Yu, D., Wanders, A., Essand, M., and Eriksson, F. 2013. An infection-enhanced oncolytic adenovirus secreting H. pylori neutrophil-activating protein 
with therapeutic effects on neuroendocrine tumors. Mol. Ther. 21(11): 2008-2018.

Streit, M., and Detmar, M. 2003. Angiogenesis, lymphangiogenesis, and melanoma metastasis. Oncogene, 22(20): 3172-3179.

Takeda, K., Kaisho, T., and Akira, S. 2003. Toll-like receptors. Annu. Rev. Immunol. 21: $335-376$.

Wang, T., Liu, X., Ji, Z., Men, Y., Du, M., Ding, C., et al. 2015. Antitumor and immunomodulatory effects of recombinant fusion protein rMBP-NAP through TLR-2 dependent mechanism in tumor bearing mice. Int. Immunopharmacol, 29(2): 876-883.

Wang, R.F., Peng, G., and Wang, H.Y. 2006. Regulatory T cells and Toll-like receptors in tumor immunity. Semin. Immunol. 18(2): 136-142.

Weidner, N., Semple, J.P., Welch, W.R., and Folkman, J. 1991. Tumor angiogenesis and metastasis--correlation in invasive breast carcinoma. N. Engl. J. Med. 324(1): 1-8.

Yin, N., Zhang, H., Luo, X., Ding, Y., Xiao, X., Liu, X., et al. 2014. IL-27 activates human trophoblasts to express IP-10 and IL-6: implications in the immunopathophysiology of preeclampsia. Mediators Inflamm, 2014: 926875.

Zhang, Y., Luo, F., Cai, Y., Liu, N., Wang, L., Xu, D., et al. 2011. TLR1/TLR2 agonist induces tumor regression by reciprocal modulation of effector and regulatory T cells. J. Immunol. 186(4): 1963-1969. 


\section{Figure Captions}

Figure 1. Treatment with rMBP-NAP protects mice against B16F10-induced metastatic lung cancer. (A) Treatment protocol. (B) Lung nodules quantification: animals from four independent experiments are represented individually. (C) The inhibition rate (IR) in response to rMBP-NAP at different doses and cisplatinum $2 \mathrm{mg} / \mathrm{kg}$ was analyzed according to the equation: $\mathrm{IR}=(1$-tumor nodules of $\mathrm{rMBP}-\mathrm{NAP} /$ cisplatinum-treated group/ tumor nodules of PBS-treated group) $\times 100 \%$. (D) Representative lung images and the mean lung weights in the tumor-bearing mice with or without rMBP-NAP treatment. (E) The body weights of each group from the first day (day 1) to the last day (day 14). (F) Survival analysis, $n=5$. Values were presented as mean \pm SEM. ${ }^{*} p<0.05,{ }^{* *} p<0.01,{ }^{* * *} p<0.001$.

Figure 2. rMBP-NAP significantly elicited systemic Th1 and cytotoxic immune response. (A) The spots pictures and statistical analysis of IFN- $\gamma$ ELISPOT assay in each group, $n=8$. (B) mRNA expression of IFN- $\gamma$ 、IL12、IL27 and IL4 in spleen. (C) The cytotoxicity of splenocytes and PBMC towards B16F10 cells was assessed using different E:T ratio. ${ }^{* *} p<$ 0.01 in comparison with the PBS group. Values were presented as mean \pm SEM. ${ }^{*} p<0.05$, ${ }^{* *} p<0.01,{ }^{* * *} p<0.001$.

Figure 3. rMBP-NAP triggered $\mathrm{T}$ cell immune response in metastatic pulmonary tumor microemviroment. (A)_and (B) The expression of Th1 like cytokines IFN- $\gamma$ and IL-27. (C) qRT-PCR analysis of mRNA expression of inflammatory cytokines and chemokines in lung tumor tissues. (D) Flow cytometric analysis of the percentage of $\mathrm{Th} 1\left(\mathrm{CD} 3^{+} \mathrm{CD} 4^{+} \mathrm{T}\right.$ cells $)$ and $\mathrm{Tc} 1\left(\mathrm{CD}^{+} \mathrm{CD}^{+} \mathrm{T}\right.$ cells) cells in single-cell suspensions of lung tissue in PBS or rMBP-NAP treated tumor-bearing mice. (E) The expression of adhesion molecules VCAM1 and ICAM1 
in lung tissue. Values were presented as mean \pm SEM. ${ }^{*} p<0.05,{ }^{* *} p<0.01,{ }^{* * *} p<0.001$.

Figure 4. rMBP-NAP inhibited melanoma metastasis tumor angiogenesis in lungs. (A) CD34 expression in lung tumor sections by immunohistochemistry; photos were taken using the inverted microscope $(200 \times$, scale bar $50 \mu \mathrm{m})$. (B) quantification of microvessel density (MVD), n=8. (C) VEGF-A and VEGF-D expression in transcriptional level by qRT-PCR. Values were presented as mean \pm SEM. . $p<0.05,{ }^{* * *} p<0.001$, difference versus control. 
(A)

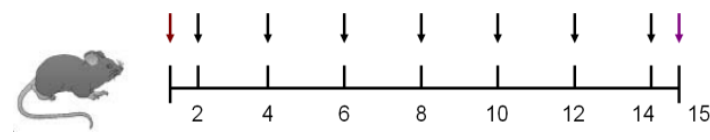

C57BL/6 mice $\downarrow$ Day 1: B16-F10 iv. (2×105cells)

$\downarrow$ Day $2,4,6,8,8,10,12,14$ i. i.p.rMBP-NAP or PBS

$\downarrow$ Day 15 : scrafice

(D)
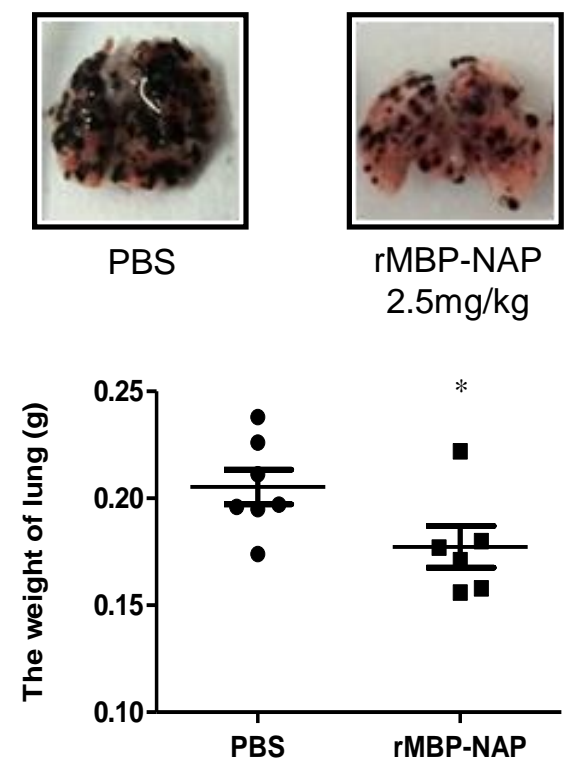

(B)

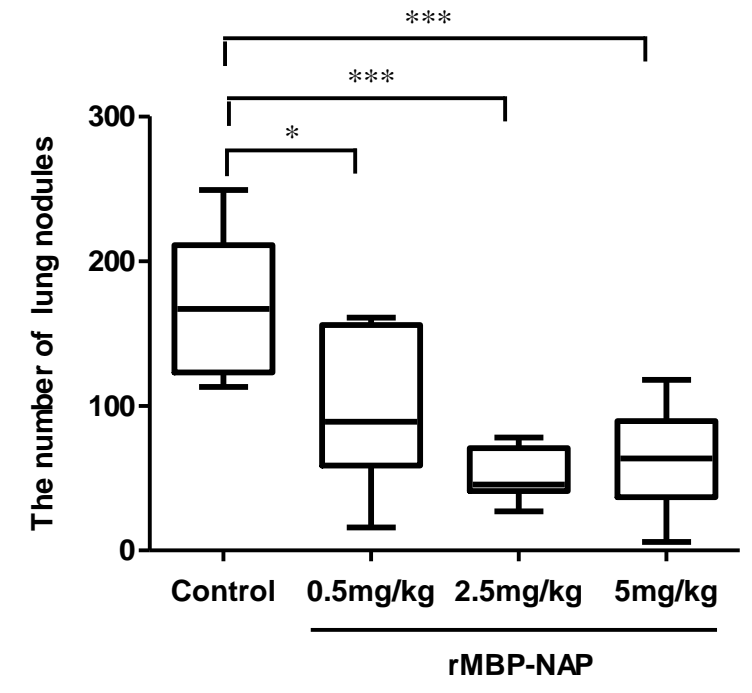

(E)

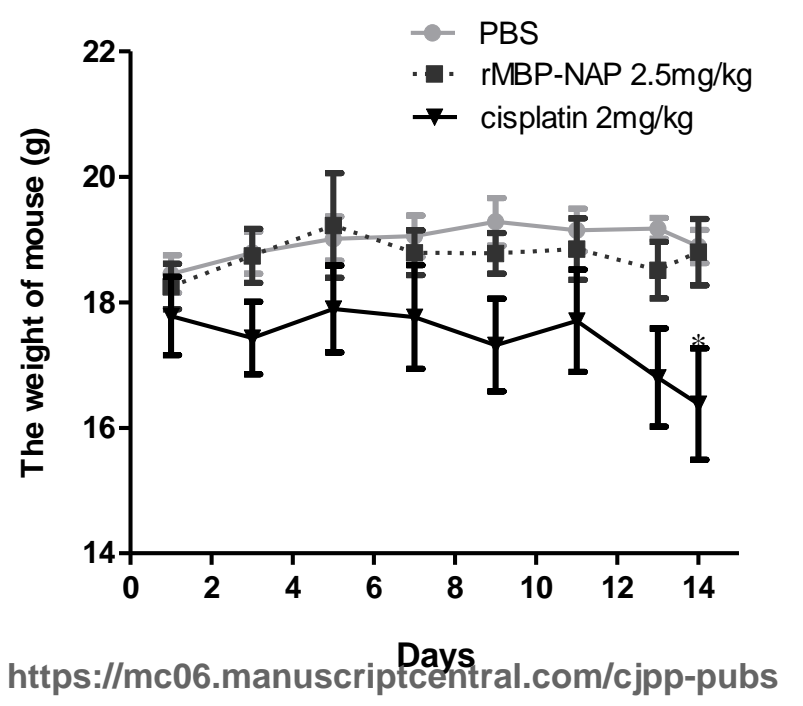

(C)

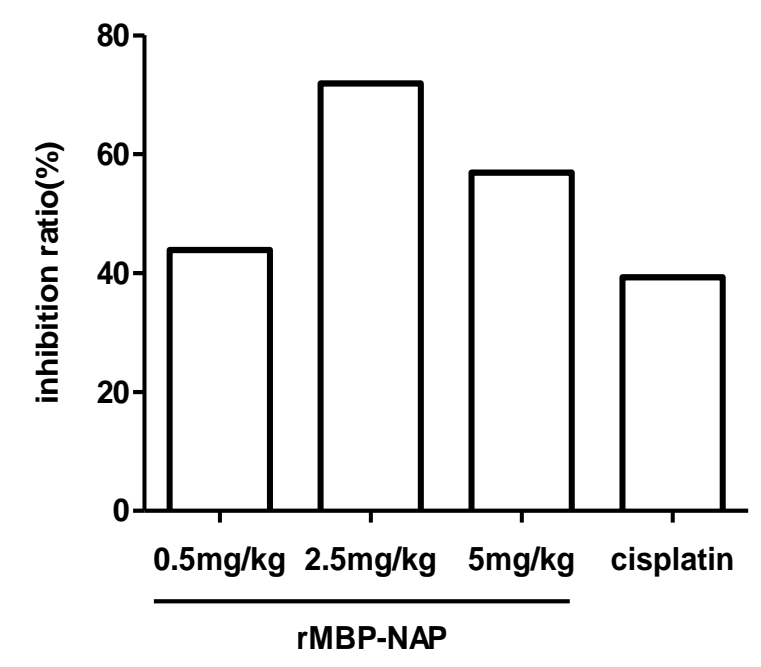

(F)

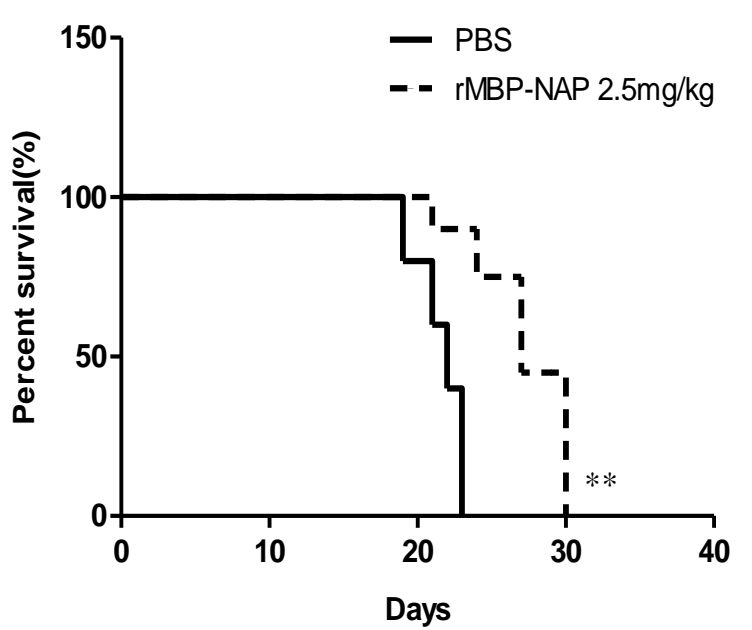


(A)

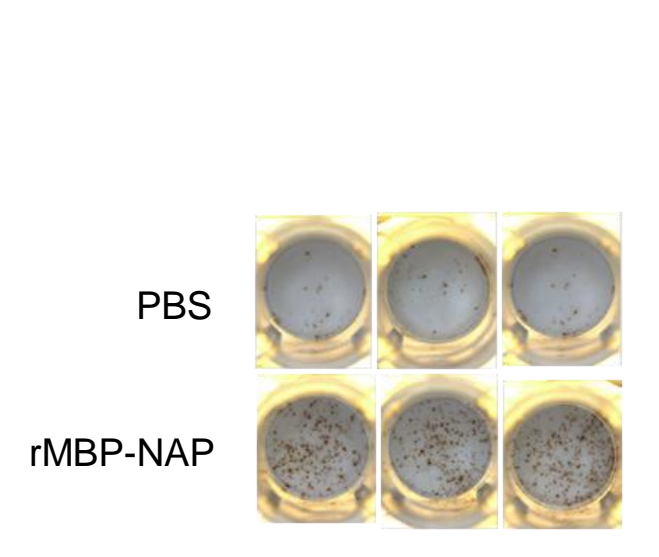

(C)

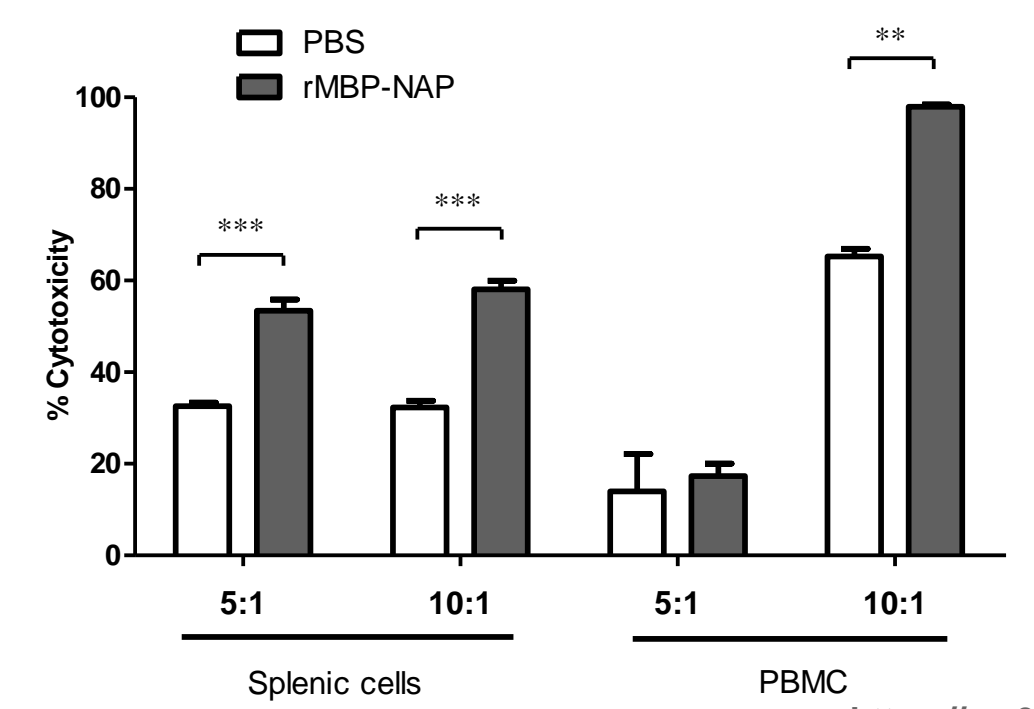

(B)

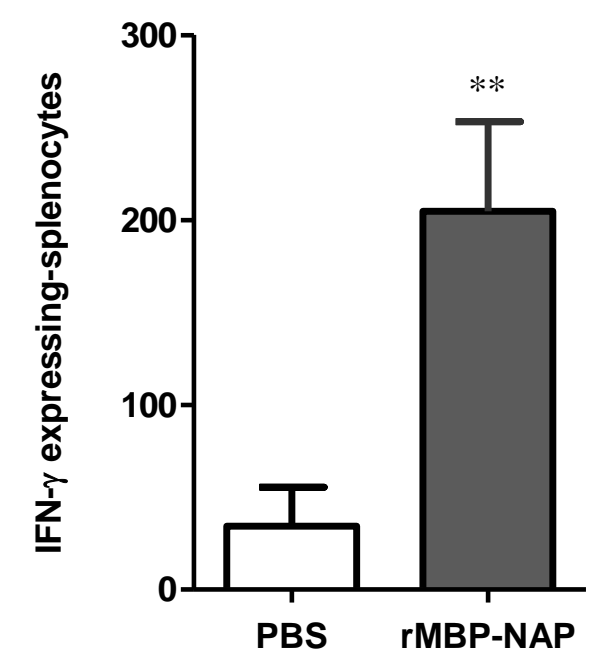

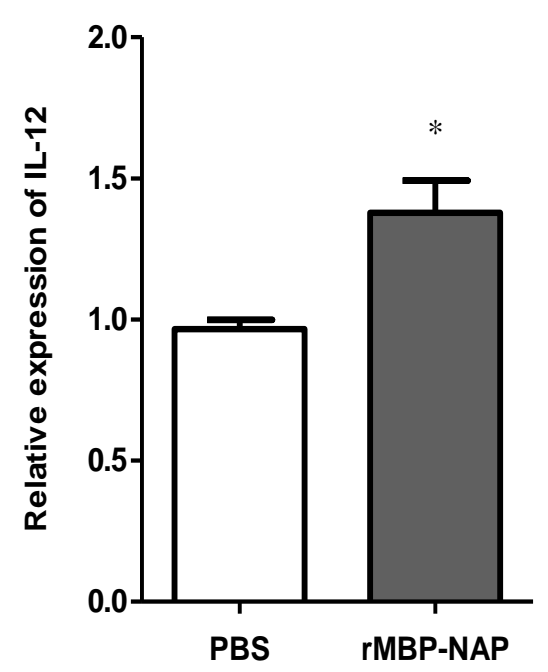

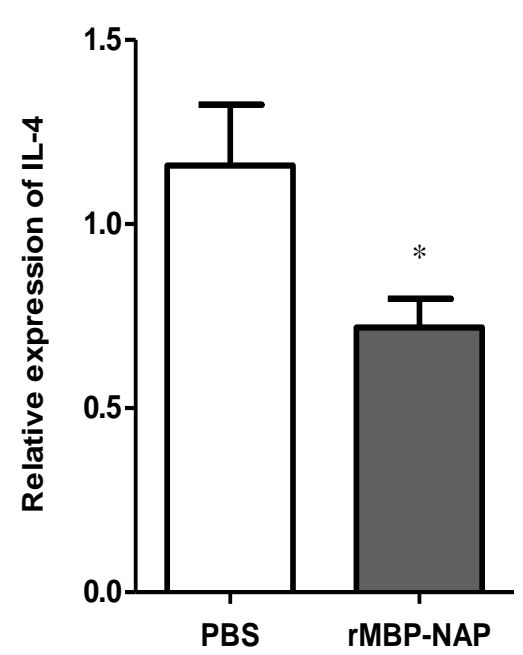


(A)

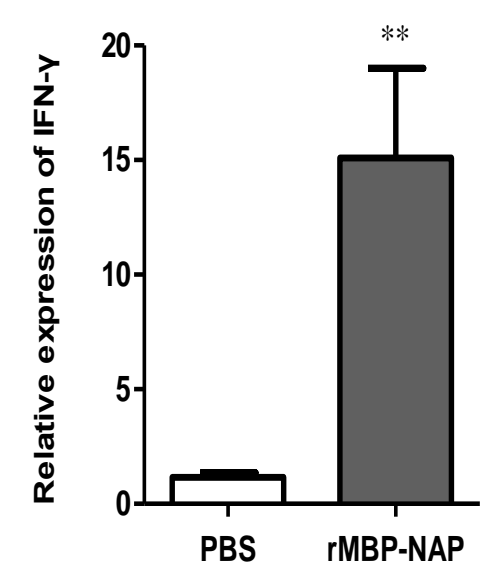

(C)

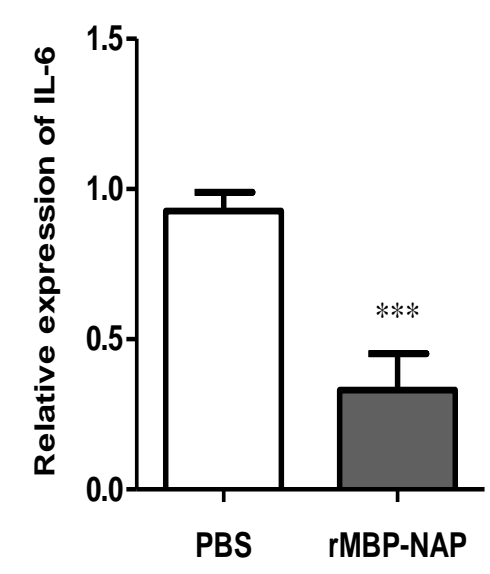

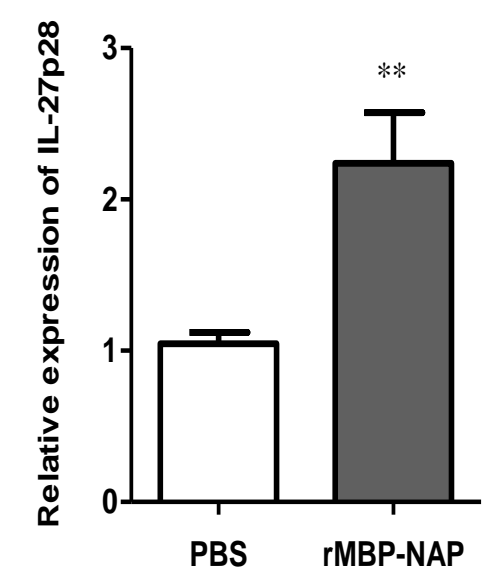

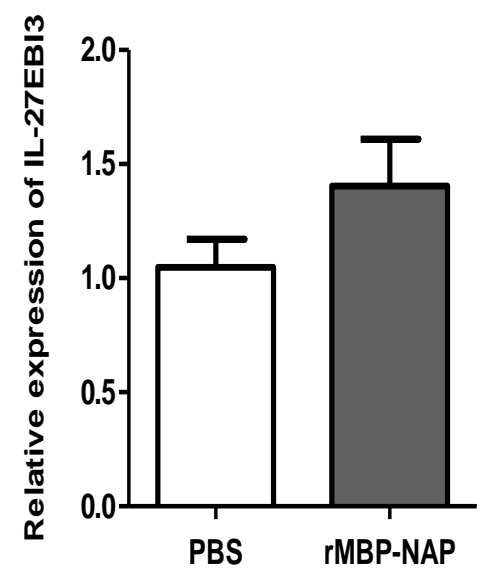

(B)
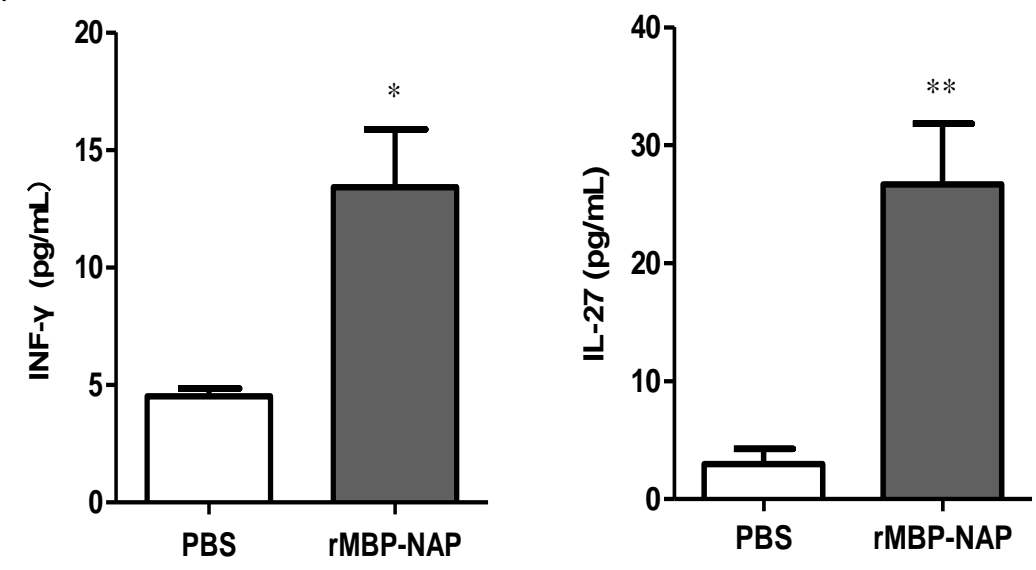
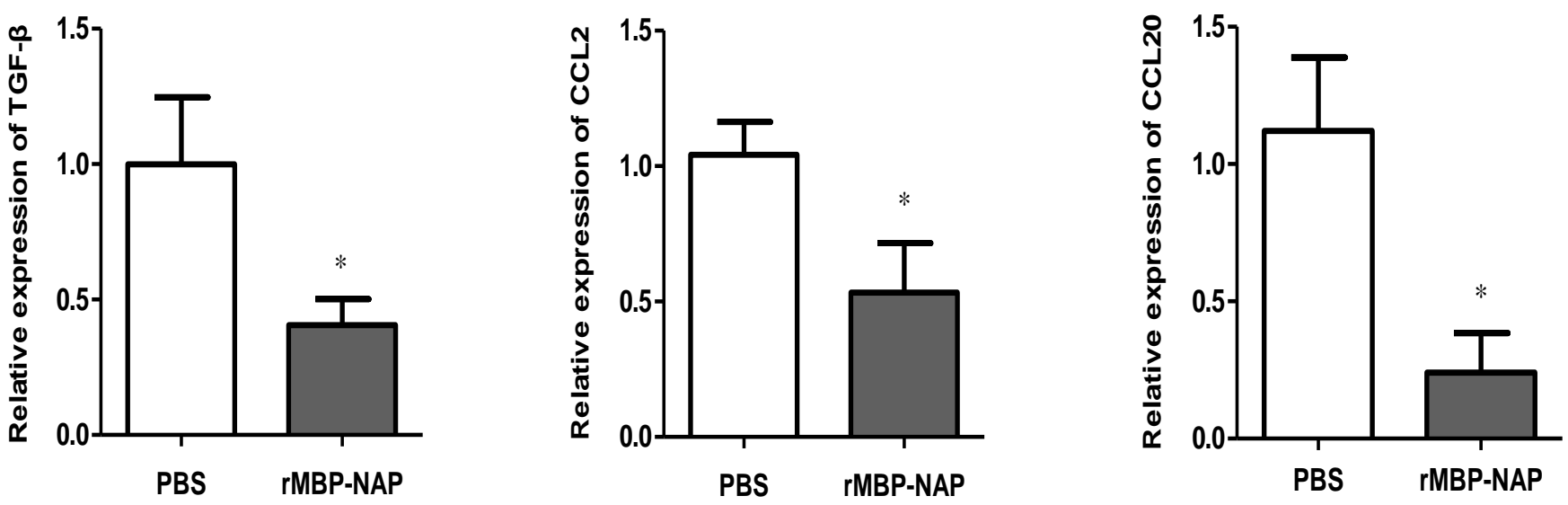
(D)
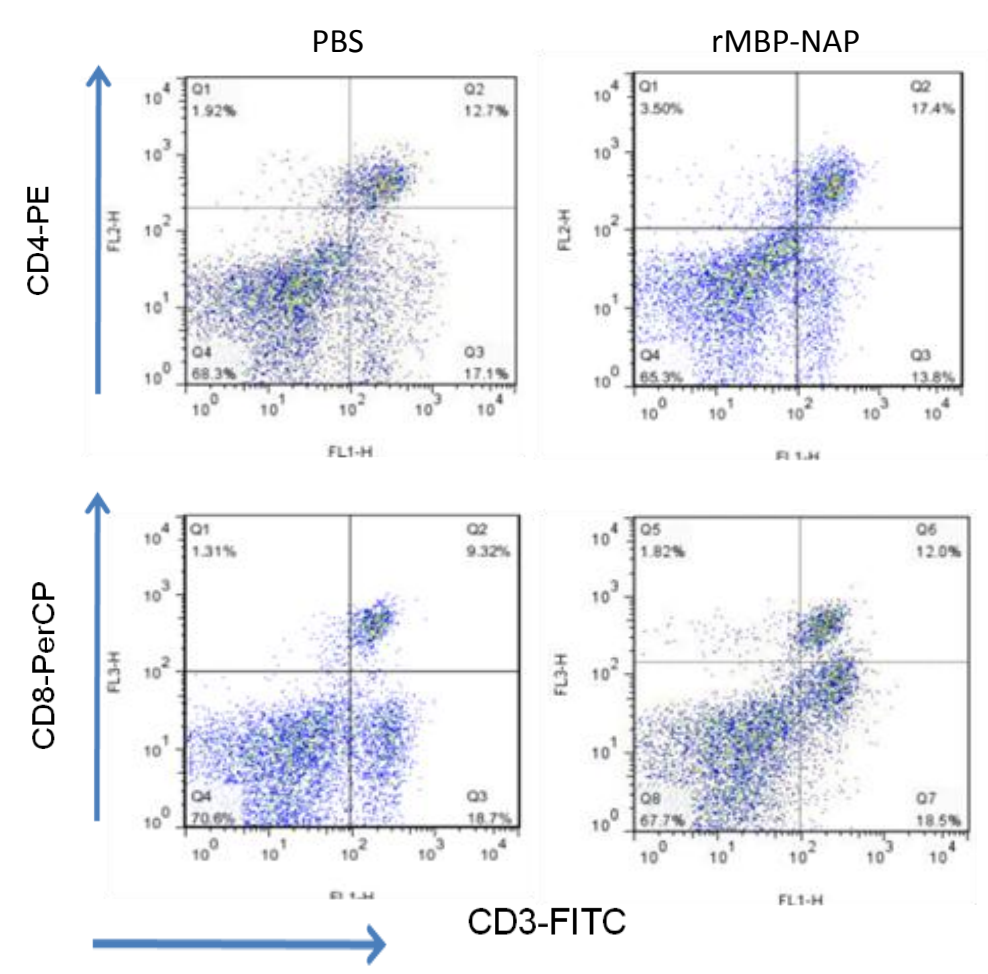
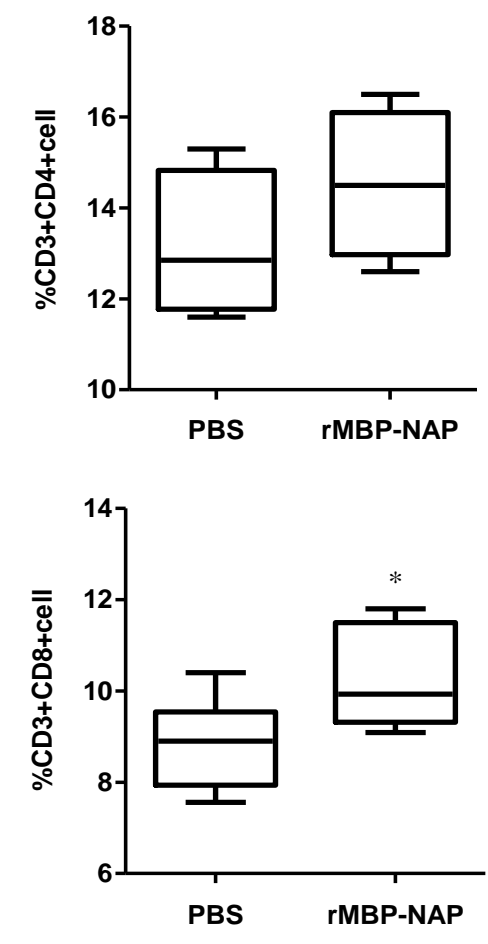

(E)
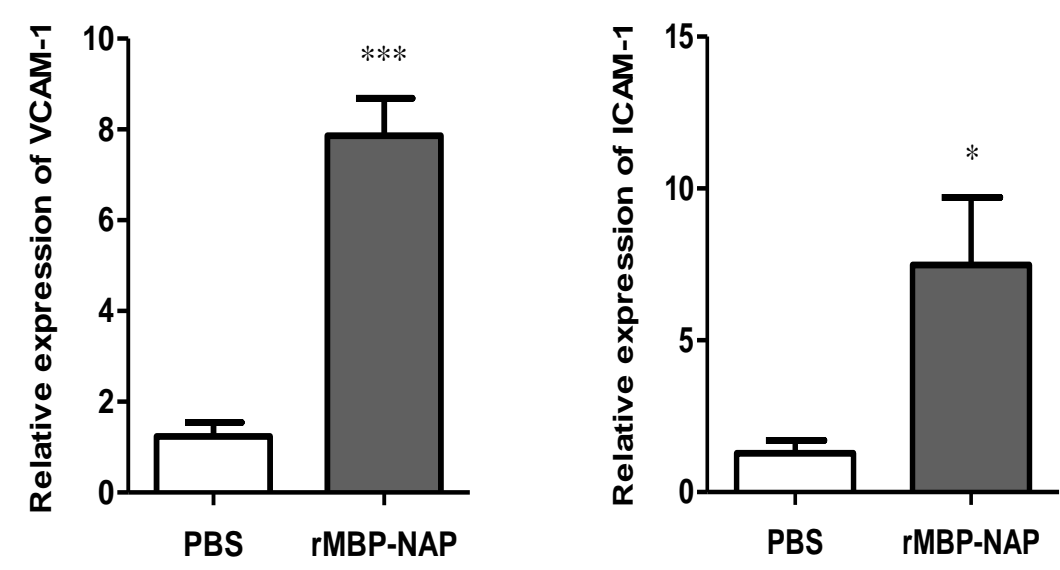
(A)
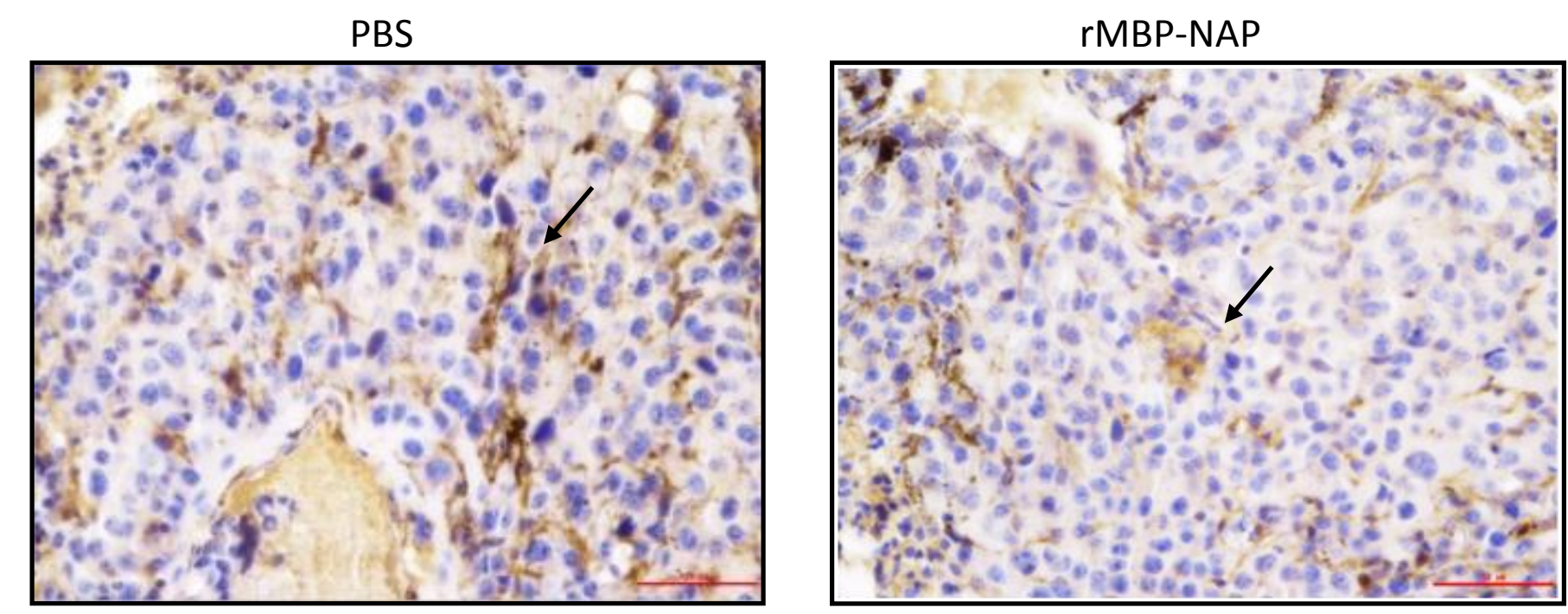

(B)

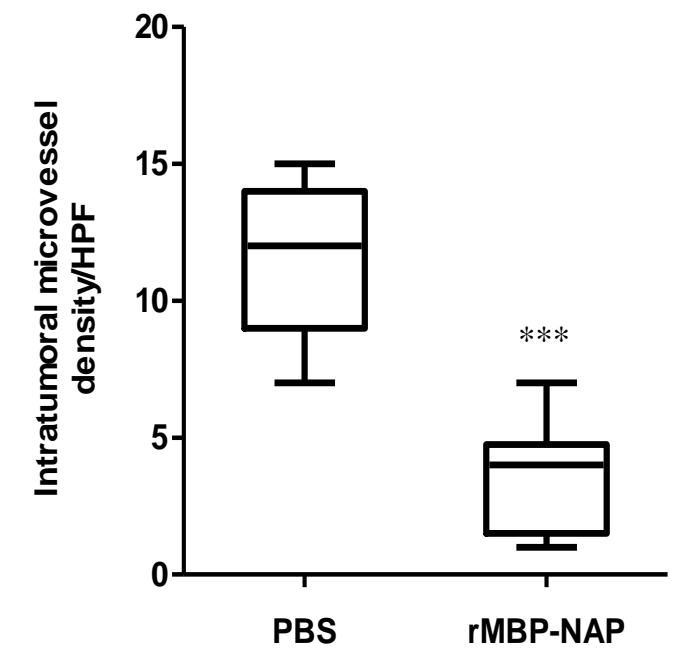

(C)

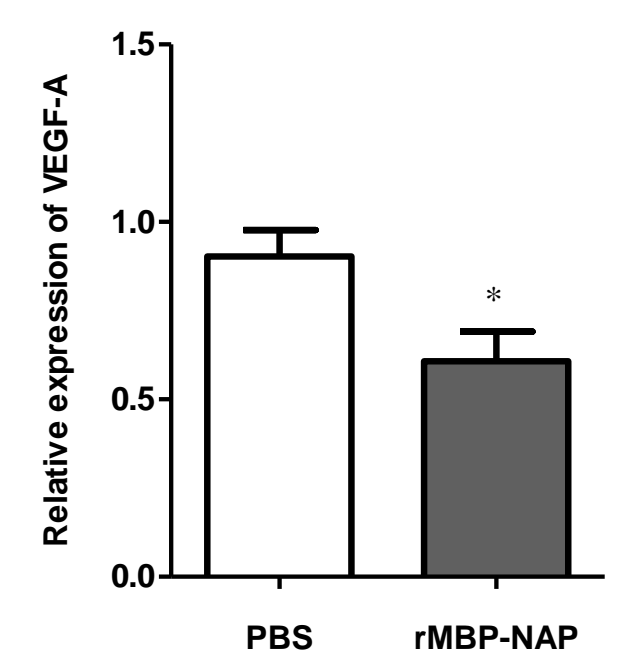

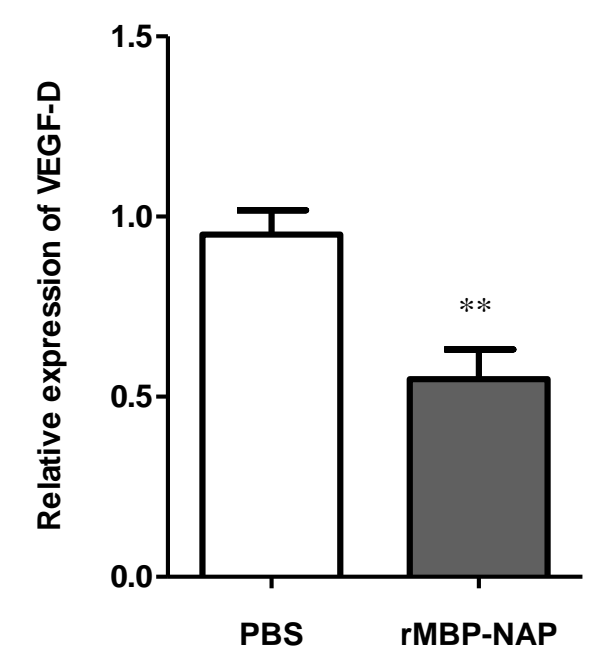

https://mc06.manuscriptcentral.com/cjpp-pubs 


\begin{tabular}{llll}
\hline Gene & Forward Primer (5) & Reverse Primer (3) & Product Size \\
\hline IFN- $\gamma$ & TCAAGTGGCATAGATGTGGAAGAA & TGGCTCTGCAGGATTTTCATG & $92 \mathrm{bp}$ \\
IL-12 & AGACATCACACGGGACCAAAC & CCAGGCAACTCTCGTTCTTGT & $77 \mathrm{bp}$ \\
IL-27p28 & TCGATTGCCAGGAGTGAACC & CGAAGTGGTAGCGAGGAAG & $127 \mathrm{bp}$ \\
IL-27EBI3 & GAGGGTCCGGCTTGATGATT & CACGGTGCCCTACATGCTAA & $101 \mathrm{bp}$ \\
IL4 & CATGGGAAAACTCCATGCTT & ATGAATCCAGGCATCGAAAA & $88 \mathrm{bp}$ \\
IL-6 & CTCTGCAAGAGACTTCCATCCAGT & GAAGTAGGGAAGGCCGTGG & $71 \mathrm{bp}$ \\
IL-10 & CCAGAGCCACATGCTCCTAGA & AGCTGGTCCTTTGTTTGAAAGAA & $75 \mathrm{bp}$ \\
TNF- $\alpha$ & AGGGTCTGGGCCATAGAACT & CCACCACGCTCTTATGTCTAC & $103 \mathrm{bp}$ \\
TGF- $\beta$ & GCTAATGGTGGACCGCAACAAC & GCACTGCTTCCCGAATGTCTG & $101 \mathrm{bp}$ \\
CCL2 & GTTGGCTCAGCCAGATGCA & AGCCTACTCATTGGGATCATCTTG & $80 \mathrm{bp}$ \\
CCL20 & CTTGCTTTGGCATGGGTACT & GAGGCAACAGTCGTAGTTGCT & $71 \mathrm{bp}$ \\
ICAM-1 & GAAGGTGGTTCTTCTGAGCG & AGGCATGGCACACGTATGTA & $370 \mathrm{bp}$ \\
VCAM-1 & AAGAGAACCCAGGTGGAGGT & GGGGCAACGTTGACATAAAG & $352 \mathrm{bp}$ \\
VEGF-A & AGCAGAAGTCCCATGAAGTGA & ATGTCCACCAGGGTCTCAAT & $82 \mathrm{bp}$ \\
VEGF-D & TCACGCTCAGCATCCCATC & ACTTCTACGCATGTCTCTCTAGG & $125 \mathrm{bp}$ \\
$\beta$-actin & GTGGCATCCATGAAACTACAT & GGCATAGAGGTCTTTACGG & $71 \mathrm{bp}$ \\
\hline
\end{tabular}

